\title{
High Resolution q-Space Imaging Studies of Water in Elastin
}

\author{
Gregory Boutis, ${ }^{1}$ Christopher Renner, ${ }^{1}$ Tamara Isahkarov, ${ }^{1}$ Tasneem Islam, ${ }^{1}$ Lahiru Kannangara, \\ Pavitarjit Kaur, ${ }^{1}$ Eugene Mananga, ${ }^{1}$ Atim Ntekim, ${ }^{1}$ Yisa Rumala, ${ }^{1}$ Doug $\mathrm{Wei}^{2}$ \\ ${ }^{1}$ Department of Earth and Physical Sciences, York College of The City University of New York, Jamaica, NY 11451 \\ ${ }^{2}$ Carl Zeiss SMT Inc., One Corporation Way, Peabody, MA 01960
}

Received 11 June 2007; revised 8 August 2007; accepted 23 August 2007

Published online 5 September 2007 in Wiley InterScience (www.interscience.wiley.com). DOI 10.1002/bip.20838

\section{ABSTRACT:}

We report on the direct measurement of the molecular diffusion coefficients of water confined to purified bovine nuchal ligament elastin by high resolution q-space NMR imaging. The experimental data indicate that water trapped within an elastin fiber has two distinguishable molecular diffusion coefficients. The component with the slowest mobility has a diffusion coefficient on the order of $10^{-6} \mathrm{~cm}^{2} / \mathrm{s}$ that varies inversely with the diffusion time and is seen to reduce near $37^{\circ} \mathrm{C}$. The component with higher mobility has a diffusion coefficient reminiscent of free water but is observed to also behave similarly at $37^{\circ} \mathrm{C}$. From our experimental data we extract the surface-to-volume ratio of pores within elastin and associated changes as a function of temperature. (1) 2007 Wiley Periodicals, Inc. Biopolymers 87: 352-359, 2007. Keywords: elastin; q-space imaging; diffusion NMR; NMR scattering

This article was originally published online as an accepted preprint. The "Published Online" date corresponds to the preprint version. You can request a copy of the preprint by emailing the Biopolymers editorial office at biopolymers@wiley. com

Correspondence to: G. Boutis; e-mail: gboutis@york.cuny.edu Contract grant sponsor: NIH/MBRS

Contract grant number: 41385-06 31

\section{(WILEY \\ InterScience}

(C) 2007 Wiley Periodicals, Inc.

\section{INTRODUCTION}

lastin is an extracellular, insoluble, and complex macromolecule that is responsible for the elasticity of many vertebrate tissues such as the skin, lung, and aorta. ${ }^{1}$ The elasticity of this remarkable biopolymer is known to be correlated to hydration since the pioneering work of Partridge. ${ }^{2}$ This has led many researchers to focus on the complex water-protein interaction by studying the thermal and mechanical properties of elastin in addition to the dynamics of repeating motifs of peptides that mimic its elasticity. Nevertheless, the mechanism resulting in the elasticity of hydrated elastin is still not well understood and several models have been put forward to account for the elastic behavior, all of which implicitly assume waters of hydration. ${ }^{3-6}$ In this work, we focus on further understanding of the complex water-elastin interaction by probing the molecular dynamics of water within elastin fibers by $q$-space NMR imaging.

Investigations of elastin and elastin-like peptides have suggested that the dynamics of water and elastin is a necessary, albeit complex, interaction for the function of the biopolymer. Calorimetric studies have suggested both a negative thermal expansion coefficient and the hydrophobic nature of the protein. ${ }^{6}$ Elastin has also been shown to undergo a glassy transition between $10^{\circ} \mathrm{C}$ and $200^{\circ} \mathrm{C}$ with a strong dependence on hydration. ${ }^{7}$ Several thermodynamic studies of elastin have indicated a strong dependence of the Young's modulus with the type of solvent and extent of hydration reinforcing the vital role of an aqueous environment that imparts its elasticity. ${ }^{8-10}$ The strain dependence of the entropy and energy components of the stress for elastin samples in swelling equilibrium with different diluents has also been investigated. ${ }^{11}$ It was shown that in water, the energetic component contributed $\sim 15 \%$ of the total stress. In DMSO, however, the energy component of the total stress of elastin was negligible in comparison to the contributing entropy component, and elastin behaved as an ideal elastomer in this solvent. While these measurements focus on 
macroscopic properties of elastin, they are all correlated to microscopic structure and dynamics of the complex protein solvent relationship.

At a molecular level, various studies also indicate the importance of hydration to the complex structure and dynamics of elastin that confers its elasticity. Recent molecular dynamics simulations have showed that $\beta$-spirals in elastin adopted more turns, distorted $\beta$-strands, buried hydrophobic residues, and main-chain polar atoms that hydrogen bonded with surrounding water. $^{12}$ Using a Boc-Gly-Leu-Gly-GlyNMe peptide, similar results were observed. ${ }^{13}$ Atomic force microscopy studies have also indicated that structures adopted by an elastin like peptide, Poly (VGGVG), in methanolic solution evolved from ribbon layers to bead filaments. In an aqueous suspension, however, the polypeptide selfassembled into a fibrillar network similar to patterns found in elastin. ${ }^{14}$ Studies of (LGGVG) ${ }_{n}$ did not adopt a single conformation lending support of a heterogeneous conformation as well. ${ }^{15,16}$ Polyalanine cross-link domains, encoded by several exons of human tropoelastin, exhibited poly-proline II helix and $\alpha$-helix conformation in aqueous and trifluoroethanol solutions, respectively. ${ }^{17}$ Several NMR dynamic studies have also pointed to elastin being highly mobile with the association of water. ${ }^{18-20}$ The NMR relaxation times of waters of hydration in elastin have been studied, and it was suggested that two distinct water components exist within elastin fibers; tightly bound waters of hydration in addition to water having more "bulk-like" properties reminiscent of free water. ${ }^{21}$ In this work, our goal is to further understand the dynamics of water within elastin fibers by $q$-space NMR imaging.

$q$-space NMR imaging is a well-known, non-invasive experimental scheme capable of probing the structure of a broad range of complex systems. ${ }^{22}$ The archetype NMR pulse sequence for these studies involves creating a spatial modulation of the magnetization, termed a grating, ${ }^{23}$ with a pulsed magnetic field gradient, and then tracking molecular displacements by measuring the attenuation of the grating due to the random motion of the molecules over an experimentally controlled time. The grating wavevector, $q$, is analogous to the neutron scattering wavevector and is determined by the relation $q=(2 \pi)^{-1} \gamma \delta g$, where $g$ is the gradient amplitude, $\delta$ is the pulsed gradient duration, and $\gamma$ is the nuclear gyromagnetic ratio. The functional form of the signal attenuation as a function of the diffusion time or gradient amplitude is determined by the geometry of the confining pore and the rate of molecular diffusion. For simple geometries, such as spherical or infinite cylindrical pores, $q$-space imaging allows for a direct measurement of the average pore diameter. When mobile molecules are confined to a porous structure, the measured diffusion coefficient will appear time dependent. In this situation, the attenuation in the signal intensity in short time allows for a measurement of the pore surface to volume ratio, independent of the pore geometry, as shown by Mitra et al. ${ }^{24}$ In the long-time regime, the time dependence in the diffusion coefficient approaches an asymptotic value allowing for a measure of the tortuosity of the system, which characterizes pore connectivity and fluid transport.

A challenging aspect of such studies is that spin relaxation causes the net magnetization to decay before the nuclear spins can "see" to full extent the complex connectivity of the system. Thus, probing the tortuosity limit in practice is experimentally difficult due to the fact that signal-to-noise is reduced by relaxation processes if the diffusion time is made too long. Mair et al. reported on a measurement of the long-time diffusion dynamics of xenon gas imbibed in glass beads allowing for a measurement of the tortuosity of this model system. ${ }^{25}$ The advantages in using a gas over a liquid are the diffusion coefficient of gas is much faster, and the relaxation times are much longer and can be controlled to some extent by adjustment of the pressure in the system. Measuring the diffusion coefficient in short time, however, is also challenging due to the fact that the gradient ringdown times and eddy currents need to be kept short. In addition, corrections for finite pulse widths are generally required as reported by Zielinski et al. $^{26} \mathrm{~A}$ detailed simulation study, recently reported by Conradi et al., examined the limits for which the surface-to-volume ratio can be determined using these schemes. ${ }^{27}$ Despite these experimental limitations, $q$-space NMR still lends a great deal of information to the study of complex systems. Latour et al. applied $q$-space NMR methods to investigate the time-dependent behavior of water diffusion in packed erythrocytes and estimated the surface-to-volume ratio and permeability of the cells. ${ }^{28} \mathrm{Q}$-space NMR is a robust technique that is in clinical use in the investigation of various neurological disorders such as Parkinson's and Alzheimer's disease.

The experimental challenge for interrogating the internal structure of an elastin fiber, and for probing the rate of molecular motion of waters of hydration by $q$-space NMR imaging, is that the magnetization grating must have a wavelength less than the diameter of the fiber. Bovine nuchal ligament elastin studied in this work has a diameter approximately equal to $5 \mu \mathrm{m} .{ }^{29}$ To create a grating with a wavelength less than $5 \mu \mathrm{m}$, strong pulsed gradient fields in excess of $1 \mathrm{~T} / \mathrm{cm}$ must be delivered to the nuclear spin ensemble that are not commercially available and were designed in our laboratory. 

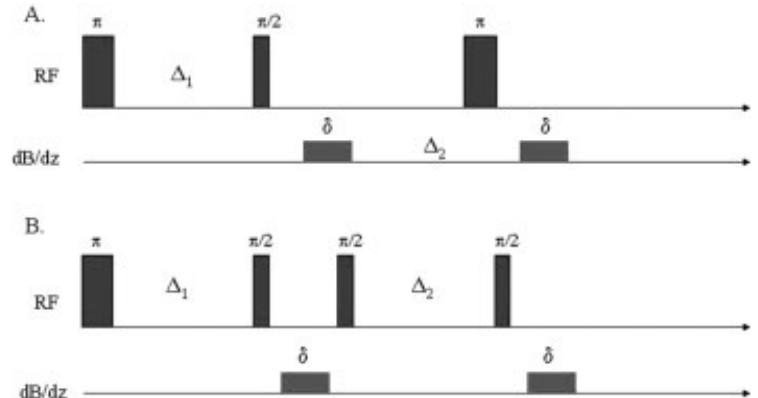

FIGURE 1 (A) Pulsed gradient spin echo (PGSE) and (B) stimulated echo (SE) pulse sequences with the inversion recovery filters. In the experiment, the time $\Delta_{1}$ is experimentally set to filter the signal arising from bulk water outside the fiber. The time $\Delta_{2}$ is the diffusion time, which was varied in the experiment. In our experiments, the amplitude of the gradient pulse is also varied, while the gradient pulse length was set to $1 \mathrm{~ms}$. The $\pi / 2$ pulse width was $3.7 \mu \mathrm{s}$, and the recycle delay was set to $10 \mathrm{~s}$ throughout all our studies.

\section{MATERIALS AND METHODS}

Purified bovine nuchal ligament samples were purchased from Elastin Products Company (Elastin Products Co., Owensville, MO) that were prepared by the neutral extraction method of Partridge. ${ }^{2}$ The samples were hydrated in deuterated water for 1 week in a mechanical shaker and included $0.03 \%(\mathrm{w} / \mathrm{v})$ of sodium azide added as biocide. The hydrated samples were placed in a sterile $10 \mathrm{~mm}$ by $1.5 \mathrm{~mm}$ OD capillary sample tube sealed with epoxy to avoid water loss. We used a modified wide-bore liquids Bruker NMR probe fitted with a home-built gradient set and high power RF capacitors. The gradient coil had a resistance of $1.5 \mathrm{Ohm}$, an impedance of $\sim 25 \mathrm{mH}$ and an efficiency of $\sim 400 \mathrm{G} / \mathrm{cm} / \mathrm{A}$. The design of such gradient coils for use in solid state NMR scattering experiments is outlined by Zhang and Cory. ${ }^{30}$ The RF coil was $\sim 2 \mathrm{~mm}$ in diameter and consisted of 5 turns of 32 AWG copper wires. We ran the experiments on a Tecmag Apollo NMR spectrometer, which controlled a Techron 5050 gradient amplifier and delivered up to $50 \mathrm{~A}$ of current. In our system, ${ }^{2} \mathrm{H}$ had a resonance frequency of $\sim 27.548 \mathrm{MHz}$. A pulsed gradient spin echo (PGSE) cycle shown in Figure 1A was used to investigate the rate of molecular diffusion of water in the elastin fiber as a function of the wave-number $q$ ranging from 0 to $1.7 \times$ $10^{4} \mathrm{~cm}^{-1}$ and diffusion times $\Delta$ ranging from 3 to $12 \mathrm{~ms}$. In addition, a stimulated echo (SE) pulse cycle shown in Figure 1B was implemented to study the rate of diffusion over longer diffusion times by varying $q$ over the same range. Both sequences incorporated a $T_{1}$ filter to suppress the signal from water outside the elastin fiber, which was determined using a saturation recovery and inversion recovery experiment. Measurements of the $T_{2}$ were performed using a CPMG cycle at every temperature and indicated the presence of water in two distinct environments within the elastin fiber. The $\mathrm{T}_{2}$ values were in good agreement with those reported by Ellis and Packer on a similar elastin sample. ${ }^{14}$ Example CMPG spectra with and without the bulk water suppression via a $\mathrm{T}_{1}$ inversion recovery filter are highlighted in Figure 2 . The $\mathrm{T}_{1}$ filter is constructed by first accurately measuring the respective $T_{1}$ relaxation times of the various components of the water within the sample. Assigning

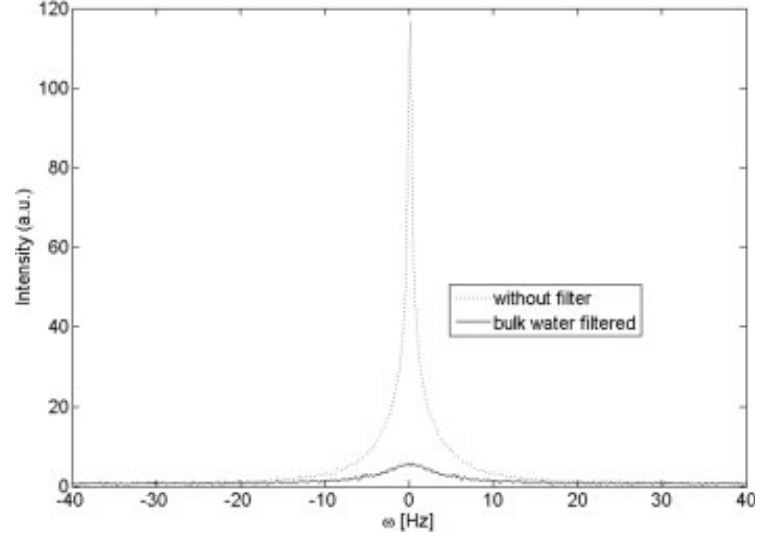

FIGURE 2 Example spectra of a free induction decay acquired with a CMPG cycle with and without the $T_{1}$ inversion recovery filter to suppress the bulk water outside the elastin fibers.

the longest $T_{1}$ to that of bulk or free water, the same $T_{1}$ inversion recovery filter was implemented in the diffusion studies.

\section{RESULTS AND DISCUSSION}

In the diffusion measurements, we found that a model of two exponentially decaying functions fit the data as $q^{2}$ was varied when the diffusion time $\Delta$ was less than $8 \mathrm{~ms}$. However, when the diffusion time was made longer a single exponential fit appeared to accurately describe our results. An example of these raw data acquired at $42^{\circ} \mathrm{C}$ for a diffusion time $\Delta$ $=2 \mathrm{~ms}$ and $\Delta=13 \mathrm{~ms}$ is shown on a logarithmic scale in Figure 3 , highlighting the signal decay as $q$ is incremented.

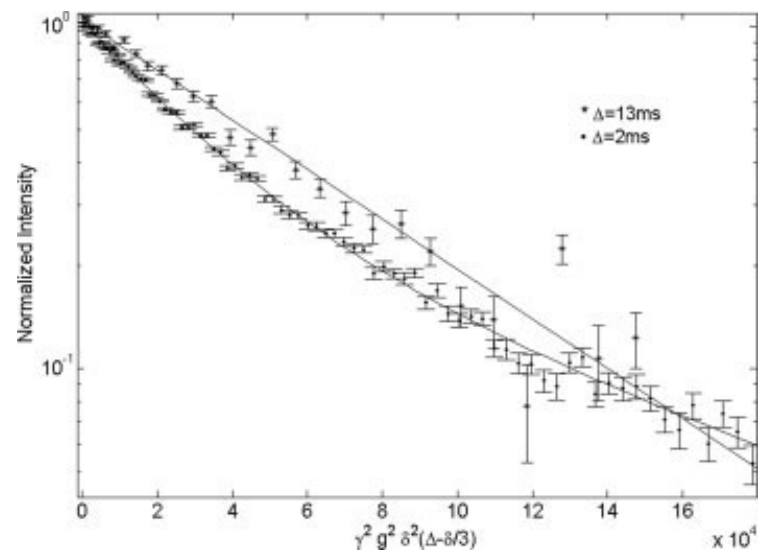

FIGURE 3 Sample experimental data acquired at $42^{\circ} \mathrm{C}$ using a PGSE cycle shown in Figure 1A. In the experimental results shown, $\Delta$ was fixed to $2 \mathrm{~ms}$ or ${ }^{\star} 13 \mathrm{~ms}$ and the gradient amplitude was systematically incremented as discussed in the text. The data show that in the short time $\Delta=2 \mathrm{~ms}$ a biexponential fit accurately describes the data well. In the longer time $\Delta=13 \mathrm{~ms}$, the data appear to be described by a linear function. Note that the data are plotted on a logarithmic scale on the $y$-axis and that the signal-tonoise is reduced for the $13 \mathrm{~ms}$ data, due to the longer diffusion time which results in greater echo attenuation. 
For short time $\Delta=2 \mathrm{~ms}$ a bi-exponential appears to fit the data well, whereas for a longer time $\Delta=13 \mathrm{~ms}$ a single exponential accurately describes the data. Yablonsky et al. have shown that a continuous distribution of diffusion constants from a PGSE measurement may produce a decay indistinguishable from a bi-exponential function. ${ }^{31}$ However, our rationale for fitting two exponentially decaying functions at short time, indicative of two diffusion coefficients, stems from the $\mathrm{T}_{2}$ observations mentioned earlier.

For any given value of $\Delta$ in either the SE or PGSE experiments, it is expected that the signal as a function of $q^{2}$ be described by an exponential or a sum of exponentials depending on the number of environments the water is exposed to. This can be understood as follows; for a porous system having an average pore size " $a$ " and a pore spacing " $b$ ", the signature of scattering events are revealed in the data only when $q \sim a^{-1}$ or $q \sim b^{-1}$. A well-known result from the theoretical treatment of $q$-space imaging is when $q \ll b^{-1}$ the echo intensity will decay exponentially. ${ }^{22}$ In our experiments, we vary $q$ over the range of $1-0.5 \mu \mathrm{m}$ and because any pore spacing within the fiber is much less than $1 \mu \mathrm{m}$, no scattering events are expected to be experimentally realized. In addition, the motion of water molecules confined in the elastin fiber is expected to take a tortuous path because of the complex morphology of the system as revealed in SEM studies. $^{32}$ Figure 4A highlights an SEM image of an intact single elastin fiber, and Figure $4 \mathrm{~B}$ shows a bovine nuchal ligament elastin fiber that was crushed under liquid nitrogen. The arrow in the figure highlights one of the many tortuous paths a water molecule can undergo. As a consequence of this tortuous path, the diffusive behavior of the water molecules in highly confined regions is expected to be time dependent due to the presence of interconnecting channels that confine their motion. Figure $4 \mathrm{C}$ highlights a cartoon whose purpose is to illustrate that the diffusion coefficient measured along the gradient axis, which in our experiment is applied along the azimuth, will be time dependent due to the tortuous path followed by the water molecules.

For such a porous system, Mitra et al. ${ }^{24}$ showed that for small $t$ that the surface-to-volume ratio can be determined from the relation

$$
\frac{D(t)}{D_{0}}=1-\frac{4}{9 \sqrt{\pi}} \frac{S}{V_{\mathrm{p}}} \sqrt{D_{0} t}+O\left(D_{0} t\right)+\cdots \quad \text { (short time) }
$$

independent of the confining geometry. In the above equation, $D_{0}$ is the free diffusion coefficient and $S / V_{\mathrm{p}}$ is the surface-to-volume ratio of the pore. For long times, the measured time dependence in the diffusion coefficients vanishes

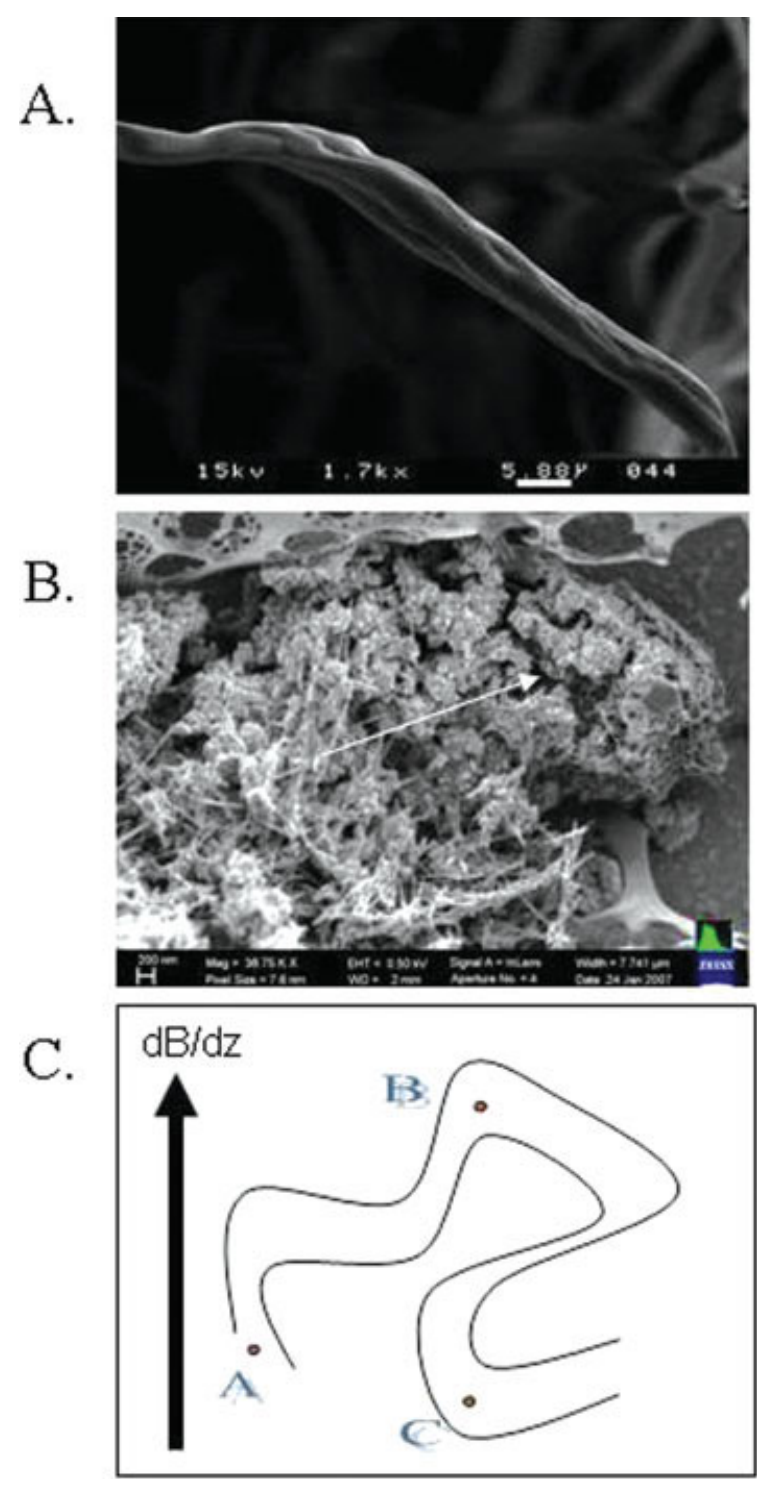

FIGURE 4 (A) SEM image of a bovine nuchal ligament fiber studied in this work. (B) High resolution SEM image of an elastin fiber crushed under liquid nitrogen. The arrow in the figure points out one of the many tortuous paths a water molecule can undergo resulting from the complex morphology of elastin. (C) A cartoon that emphasizes that the measured diffusion coefficient along the azimuthal axis will appear time dependent and reduced as a molecule moves from A to B in a time $t 1$, compared with one displacing a distance $\mathrm{A}$ to $\mathrm{C}$ in a longer time $\mathrm{t} 2>\mathrm{t} 1$.

and the diffusion coefficient is related to the inverse of the tortuosity of the confining channel denoted by $\alpha$. That is,

$$
\frac{D(t)}{D_{0}}=\frac{1}{\alpha}+\frac{c}{t}+O\left(\frac{1}{t}\right)^{3 / 2}+\cdots(\text { long time })
$$

where $c$ is a constant that is again determined by the morphology of the system. To analyze the intermittent region between short-time and long-time behaviors, a Padé approxi- 


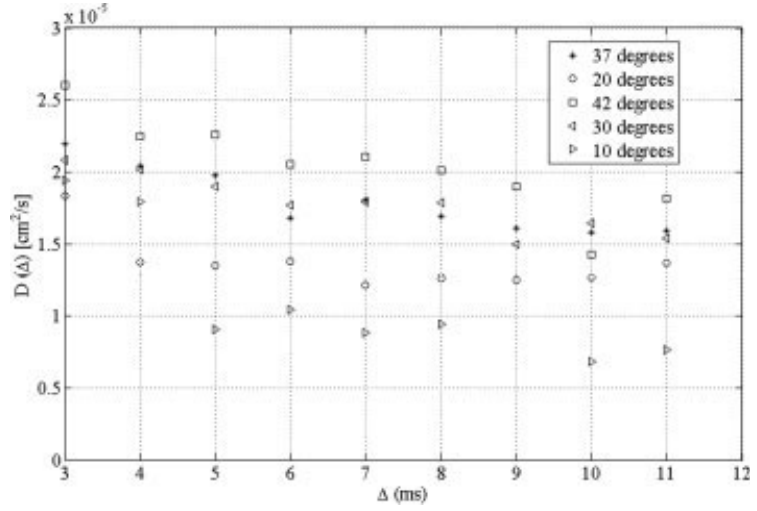

FIGURE 5 Compiled diffusion coefficients of the component of water having bulk-like properties, by virtue of the magnitude of the diffusion coefficient, in elastin fibers as a function of temperature and the diffusion time measured by a PGSE cycle. In the text, this component is referred to as the $\beta$ component. The errors in the data are $\sim 5-10 \%$ of the value shown.

mation was introduced by Latour to extrapolate between these two regimes which is written

$$
\begin{aligned}
\frac{D(t)}{D_{0}}=1-\left(1-\frac{1}{\alpha}\right) \frac{4 \sqrt{D_{0} t} S}{9 \sqrt{\pi V_{\mathrm{p}}}} & \\
& +\frac{\left(1-\frac{1}{\alpha}\right) \frac{D_{0} t}{D_{0} \theta}}{\left(1-\frac{1}{\alpha}\right)+\frac{4 \sqrt{D_{0} t} S}{9 \sqrt{\pi V_{\mathrm{p}}}}+\left(1-\frac{1}{\alpha}\right) \frac{D_{0} t}{D_{0} \theta}}
\end{aligned}
$$

In the above expression, $\theta$ has dimensions of time and represents the time molecules that diffuse an average macroscopic distance $l$ to reach the tortuosity limit. ${ }^{33}$

One final point should be noted regarding the background susceptibility effects in our measurements. These local susceptibility effects create additional gradients, which are superimposed on the applied pulsed gradient field. From our CPMG experiments, we and others ${ }^{14}$ found that the shortest $\mathrm{T}_{2}$ was on the order of $10 \mathrm{~ms}$. The shortest $\mathrm{T}_{2}^{*}$ component of the water within the elastin fibers as measured by a single pulse experiment was on the order of $200 \mu$ s. Given that the sample tube had an inner diameter of $1.5 \mathrm{~mm}$, for ${ }^{2} \mathrm{H} \gamma=$ $653 \mathrm{~Hz} / \mathrm{G}$ an estimate of the average background gradient is on the order of $50 \mathrm{G} / \mathrm{cm}$. However, because the magnitude of the pulsed gradient field imparted on the spin system is on the order of $10^{4} \mathrm{G} / \mathrm{cm}$, these local susceptibility effects are considered negligible and will not be discussed further.

Figure 5 highlights the measured values of the component of the water having a diffusion coefficient order $10^{-5} \mathrm{~cm}^{2} / \mathrm{s}$, hereafter referred to as the $\beta$-component, as a function of temperature and diffusion time. Referring to Figure 5 , the results show that the measured $\beta$-diffusion coefficient does not vary significantly as the diffusion time is changed from 4 to $12 \mathrm{~ms}$. Within the error bar of $5-10 \%$ (which were not shown for the purposes of simplifying the representation of the data), the results indicate that the $\beta$-diffusion coefficients vary only $\sim 10 \%$ over the time scale shown. From these results, we can deduce the tortuosity of the elastin in regions of the fiber where the water has bulk like properties. The early time dynamics shows slight time dependence. We believe this small variation comes from the $c / t$ dependence shown in Eq. (2). In addition, the results show that the measured diffusion coefficients near $37^{\circ} \mathrm{C}$ are very close to that observed at $30^{\circ} \mathrm{C}$ despite the increase in temperature. These findings suggest that the measured bulk tortuosity of the system is the same despite the increase in temperature, and that the expected increase in the diffusion constant due to the increase in temperature is offset by the negative thermal expansion coefficient of elastin.

In Figure 6, the measured diffusion coefficient of the water having a magnitude on the order of $10^{-6} \mathrm{~cm}^{2} / \mathrm{s}$, hereafter referred to as the $\gamma$-component, is seen to depend both on the diffusion time and on temperature. In our experiments, we observe changes in the diffusion coefficients over time scales of milliseconds, and the RMS displacement of the ${ }^{2} \mathrm{H}$ nuclei in a time $\Delta \sim 4 \mathrm{~ms}$ with $D \sim 10^{-6} \mathrm{~cm}^{2} / \mathrm{s}$ is on the order of $1 \mu \mathrm{m}$. Hence, the observed time dependence in the diffusion coefficients of the $\gamma$-component is due to the tortuous path that the water molecules undergo as discussed earlier, and the slope of the curve in short time is related to the $S / V_{\mathrm{p}}$ ratio of the channel or pore that confines the motion. In each of the graphs, we show the measured diffusion coefficient $D(\Delta)$ as a function of the square root of the diffusion time $\Delta$. The line shown in each graph is a fit to the experimental data using Eq. (1), whose slope is the $S / V_{\mathrm{p}}$ of the channel that confines the motion of the molecules. The line fit to the $10^{\circ} \mathrm{C}$ and $20^{\circ} \mathrm{C}$ data made use of all the experimental data points shown. For the higher temperatures, we used only the first 4 points in the decay of the diffusion coefficient, which appeared to have a linear behavior with $\Delta^{1 / 2}$. An important point that should be emphasized in the fitting of Eq. (1) to the experimental data is that the diffusion coefficient at $\Delta=0$ is that of free water, which is well known. The graph shows that the fit appears to describe the trend in the experimental data for the $10^{\circ} \mathrm{C}$ and $20^{\circ} \mathrm{C}$ quite well, however, not for the $30^{\circ} \mathrm{C}, 37^{\circ} \mathrm{C}$, and $42^{\circ} \mathrm{C}$ data. The physics of these results is at the $10^{\circ} \mathrm{C}$ and $20^{\circ} \mathrm{C}$ temperatures, the morphology of the elastin sample is such that the highly confined $\gamma$ water molecules traverse a distance where they sample the shape of the confining pore only, and that the tortuosity limit is not reached. The measured surface-to-volume ratio at these temperatures was $1.14 \times 10^{4} \mathrm{~cm}^{-1}$ and $1.04 \times 10^{4} \mathrm{~cm}^{-1}$ for $10^{\circ} \mathrm{C}$ and $20^{\circ} \mathrm{C}$, respectively. 

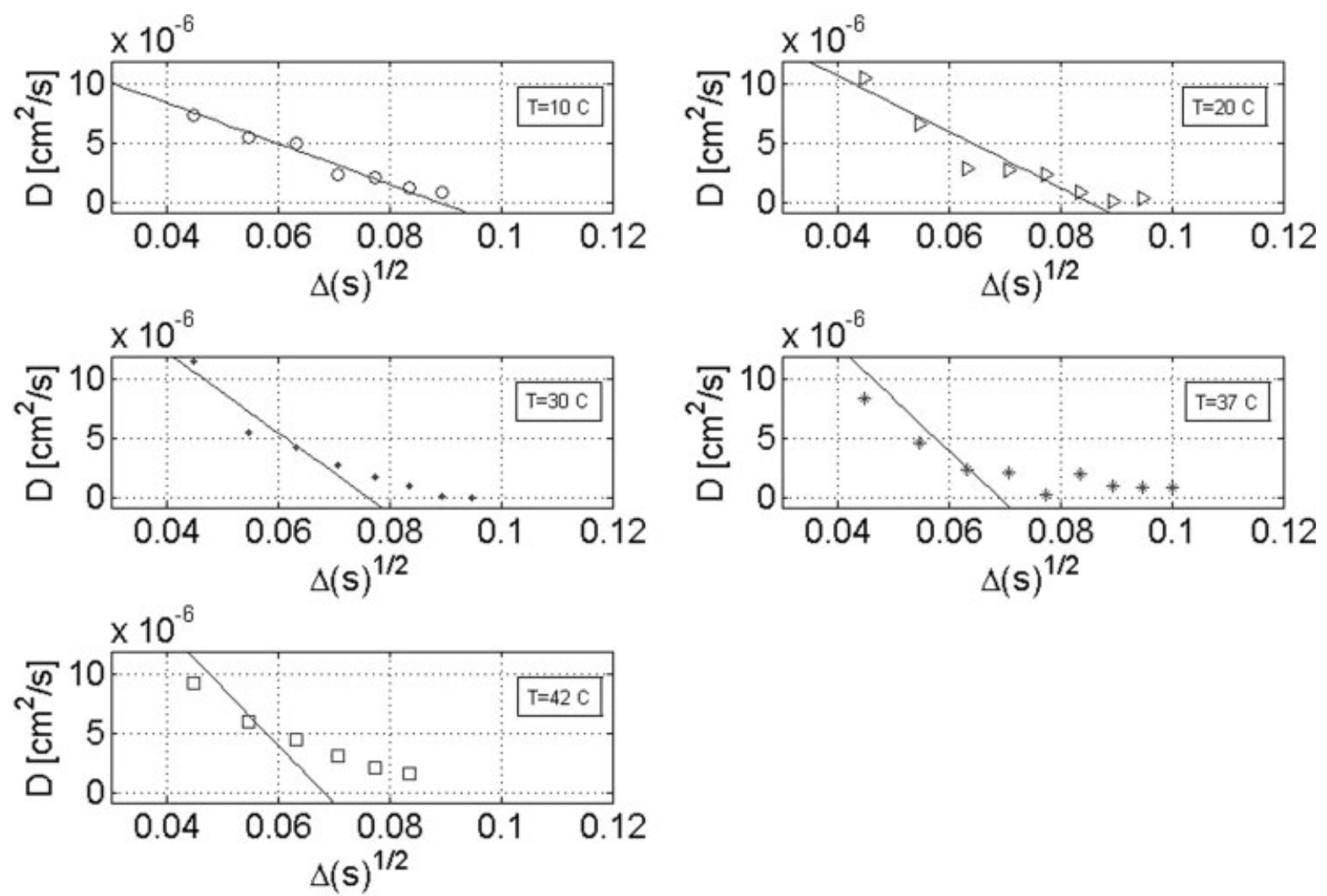

FIGURE 6 Compiled diffusion coefficients of the component of water having a magnitude on the order of $10^{-6} \mathrm{~cm}^{2} / \mathrm{s}$ in elastin fibers as a function of temperature and the square root of the diffusion time measured by a PGSE cycle. In the text, this component is referred to as the $\gamma$ component. The figures show a fit using Eq. (1) from which we extracted the surface to volume ratio of the pore or channel, which confines the motion of the molecules. The errors in the data are $\sim 5-$ $10 \%$ of the value shown.

At $30^{\circ} \mathrm{C}, 37^{\circ} \mathrm{C}$, and $42^{\circ} \mathrm{C}$ we could not fit Eq. (1) to the all of the experimental data that is shown, suggesting that the dynamics we probed are no longer satisfying the short-time approximation, which is tacitly implied in this expression. Careful inspection of $D(\Delta)$ at $30^{\circ} \mathrm{C}$ and $42^{\circ} \mathrm{C}$ reveals that the results also show a time dependence at long times from which we deduce that tortuosity limit has not been reached with these measurements. Moreover, the shape of the curve appears to vary in going from 30 to $37^{\circ} \mathrm{C}$ and then to $42^{\circ} \mathrm{C}$. The Padé approximation given in Eq. (3) extrapolates between Eqs. (1) and (2) and requires an estimate of the $S / V_{\mathrm{p}}$ ratio and the tortuosity of the system in order for the parameter $\theta$ to be measured. In an ideal experimental condition, we would have shortened the observation time $\Delta$ below $1 \mathrm{~ms}$ and probed the short time dynamics of the molecules and more accurately measured $S / V_{\mathrm{p}}$. Currently, the gradient ringdown times and eddy currents in our setup prevents us from doing so. In addition, if the $\mathrm{T}_{2}$ were sufficiently long, we would also probe longer time dynamics of the water and measure $\alpha$, the tortuosity. Together, $\alpha$ and $S / V_{\mathrm{p}}$ would have been used to fit Eq. (3) to our data to estimate $\theta$.

In an attempt to study the dynamics and measure the tortuosity of the $\gamma$-component in the sample in the long-time regime, we implemented a SE pulse sequence. The results obtained when $\Delta=25,50$, and 75 ms indicated only 1 diffusion coefficient, and the values we measured are shown in Figure 7 as a function of temperature. We find that the measured values are slightly above those measured by the PGSE cycle for the $\gamma$-component shown in Figure 6 . At $10^{\circ} \mathrm{C}$ the diffusion coefficient measured with $\Delta=8 \mathrm{~ms}$ with a PGSE experiment was $8.6 \times 10^{-7} \mathrm{~cm}^{2} / \mathrm{s}$, but in the SE experiment we measured $\sim 1.4 \times 10^{-6} \mathrm{~cm}^{2} / \mathrm{s}$ at $\Delta=25,50$, or $75 \mathrm{~ms}$. We believe these results may suggest that over the time scale of $25-75 \mathrm{~ms}$ that the highly confined $\gamma$-component exchanges with the bulk-like $\beta$-component. A consequence of this exchange prevents us from precisely measuring the tortuosity limit of the $\gamma$-component at all temperatures. While further experimental work is necessary to quantify this exchange, 


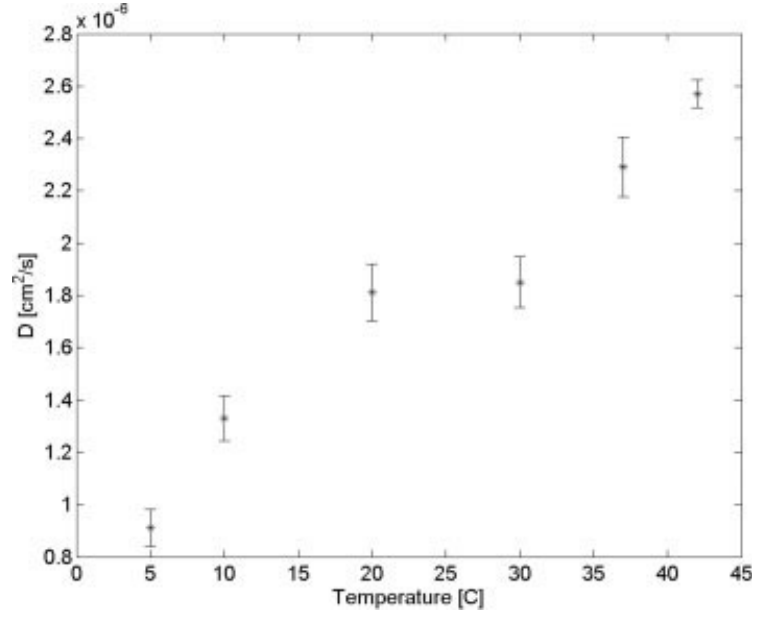

FIGURE 7 Measured diffusion coefficients using a SE cycle as a function of temperature. The measured diffusion coefficient represents the average values from experiments performed where $\Delta_{2}$ was 25,50 , and $75 \mathrm{~ms}$.

here we provide an estimate of the $S / V_{\mathrm{p}}$ ratio from the first few points in the decay for the temperatures above $20^{\circ} \mathrm{C}$. The measured values are $1.02 \times 10^{4} \mathrm{~cm}^{-1}, 1.04 \times 10^{4} \mathrm{~cm}^{-1}$, and $1.01 \times 10^{4} \mathrm{~cm}^{-1}$ for $30^{\circ} \mathrm{C}, 37^{\circ} \mathrm{C}$ and $42^{\circ} \mathrm{C}$, respectively.

The changes in the shape of the diffusion coefficient versus the diffusion time curve shown in Figure 6 may point to morphology changes within the elastin fiber as the temperature is varied. At the lower temperatures the data appear to be well described by a straight line, in agreement with the leading term of Eq. (2). However, at temperatures above $20^{\circ} \mathrm{C}$ we find that the diffusion coefficients as a function of $\Delta^{1 / 2}$ are no longer linear as measured over the same time scale. Recalling again that elastin has a negative thermal expansion coefficient, an increase in temperature decreases the volume of the entire fiber. If we very crudely model the pore that inhibits the motion of the $\gamma$ water molecules as a sphere of radius $R$, then the surface to volume ratio is given by $3 / R$. Hence, a reduction only in the radius $R$ of the pore would increase the $S / V_{\mathrm{p}}$ as the temperature is raised. Our results do not indicate a significant change in the measured $S / V_{\mathrm{p}}$ as the temperature is raised. The fact that we observe changes in the shape of the diffusion curve as a function of the square root of the diffusion time suggests a complex change in the average micro-pore area and volume within the elastin fiber or that the connectivity of the channels within the elastin fiber may change as a function of temperature. In addition, the data also suggest that the slope of the curve changes between 4 and $8 \mathrm{~ms}$ in going from 30 to $42^{\circ} \mathrm{C}$. More specifically, at $37^{\circ} \mathrm{C}$ we find that the shape of the curve appears to have reached an asymptotic value. Recalling that the asymptotic value of the diffusion coefficient in long time is a measure of the tortuosity of the system, we estimate $\alpha=$ 33.0 for the $\gamma$ water molecules in elastin at the $37^{\circ} \mathrm{C}$, suggesting a highly complex interconnected geometry, as illustrated in the crushed fiber shown in Figure $4 \mathrm{~B}$.

The fact that the water near $37^{\circ} \mathrm{C}$ within elastin fibers appears to approach an asymptotic value fast than other temperatures, appears to also be correlated with more microscopic dynamics of the protein as reported by others. The work reported by Fleming et al. showed that the line-width of the carbonyl peak of a ${ }^{13} \mathrm{C}$ labeled, hydrated aortic elastic sample is maximum near $32^{\circ} \mathrm{C} .{ }^{19}$ At both lower and higher temperatures, the line-widths are narrower than that at $32^{\circ} \mathrm{C}$. A well known result from NMR is that a wide line-width is indicative of slow molecular motion, ${ }^{22}$ so that the observed broadening of the ${ }^{13} \mathrm{C}$ carbonyl peak at $32^{\circ} \mathrm{C}$ is indicative of reduced motion relative to both higher and lower temperatures. A similar trend was observed by Perry et al. in their investigation of a ${ }^{13} \mathrm{C}$ labeled elastin sample; the linewidth of the carbonyl on the protein backbone increased and peaked at $37^{\circ} \mathrm{C}$ for a fully hydrated sample as the temperature was increased from -20 to $37^{\circ} \mathrm{C} .{ }^{20}$ Together, the reduction in the motion of the protein backbone at $37^{\circ} \mathrm{C}$, and the negative thermal expansion coefficient results in water molecules in the highly confined regions of elastin to more rapidly reach the tortuosity limit near $37^{\circ} \mathrm{C}$ than at any of the lower temperatures. Careful investigation of the data of the $\gamma$ component at $42^{\circ} \mathrm{C}$ reveals that the diffusion coefficients are again slightly greater than that measured at $37^{\circ} \mathrm{C}$, while the sample would have further decreased its volume due to the negative thermal expansion coefficient. For the same diffusion times, the tortuosity limit is not reached at $10^{\circ} \mathrm{C}$ and $20^{\circ} \mathrm{C}$ as observed at higher temperatures. Together with the spectroscopic data mentioned earlier, the diffusion data appear to indicate that the microscopic changes in the morphology and dynamics of the protein tightly confine the water molecules that are in close proximity near the physiological temperature.

The highly confined water and the associated reduction in the volume of the interstices, which confine their motion at the physiological temperature, may play a role in the elasticity of elastin. The results of Mistrali et al. indeed showed that water contributes a non-negligible amount to the strain dependence of the energy components of the stress. ${ }^{11}$ Though the protein itself must withstand the mechanical strain when an externally applied mechanical force is applied, the surface tension within a water filled pore may play a role in the restoring force giving rise to elasticity observed only in hydrated samples. Considering the thermodynamic definition of the surface tension, $\gamma=\partial W / \partial S$, where $W=P \partial V$ is the mechanical work applied to the system, and $S$ is the area over which the work is applied, the surface tension may be 
rewritten as $\gamma=P \partial V / \partial S$. Water, or any other polar solvent, confined within micro-pores of an elastin fiber that undergoes a deformation in the ratio of the volume-to-surface ratio, $\partial V / \partial S$, may contribute a non-negligible surface tension. One can extend the scattering studies presented here to probe this surface tension within an elastin fiber by measuring changes in the $S / V_{\mathrm{p}}$ of the pores, while a well-defined mechanical stress is applied to the sample.

Russo et al. recently reported on an experimental study of hydration dynamics near a model hydrophobic amino acid by neutron scattering and by simulation. ${ }^{34}$ Their work showed that the diffusion coefficients of water surrounding the model hydrophobic system, both in the bulk and in close proximity to the hydrophobic amino acid, may be reduced by approximately 1 order of magnitude relative to free water as realized in the experimental results presented here. In simulation studies, the residence time of water molecules near elastin is on the order of ps and water molecules in the hydration shells appear to be more structured at $10^{\circ} \mathrm{C}$ compared with $42^{\circ} \mathrm{C}$. ${ }^{12}$ Our results do not separate out the different hydration shells that surround elastin, because we study the long-time behavior over a time scale of tens of milliseconds.

\section{CONCLUSIONS}

In this work, we report on the direct measurement of the rates of molecular diffusion of waters of hydration confined to purified bovine nuchal ligament elastin fibers, via a $q$ space NMR scattering experiment. The experimental data indicate two distinguishable diffusion coefficients allowed for a measurement of the average $S / V_{\mathrm{p}}$ ratio of the pores and the associated tortuosity at the physiological temperature. With stronger gradients than that presented here, it is expected that one may probe scattering events within micropores of single elastin fibers to further elucidate the complex waterprotein interaction.

The authors thank Professor R. Mecham from Washington University, Gabor Borzsonyi from the University of Alberta for useful discussions, and Doug Wei of Carl Zeiss for helping us generate the SEM images of the elastin fiber. This publication was made possible by Grant Number 41385-06 from the National Institute of Health/ MBRS and by a grant from The City University of New York PSCCUNY Research Award Program.

\section{REFERENCES}

1. Linsenmeyer, T. F. Cell Biology of Extracellular Matrix, 2nd ed.; Plenum: New York, 1991, pp 4-44.
2. Partridge, S. M.; Davies, H. F.; Adair, G. S. Biochem J 1955, 61, 11-21.

3. Hoeve, C. A.; Flory, P. J. Biopolymers 1974, 13, 677-686.

4. Weis-Fogh, T.; Andersen, S. O. Nature 1970, 227, 718-721.

5. Gray, W. R.; Sandberg, L. B.; Foster, J. A. Nature 1973, 246, 46-466.

6. Venkatachalam, C. M.; Urry, D. W. Macromolecules 1981, 14, 1225-1229.

7. Kakivaya, S. R.; Hoeve, C. A. Natl Acad Sci 1975, 72, 3505-3507.

8. Lillie, M. A.; Gosline J. M. Int J Biol Macromol 2002, 30, 119127.

9. Lillie, M. A.; Gosline J. M. Biopolymers 1995, 39, 641-652.

10. Lillie, M. A.; Gosline, J. M. Biopolymers 1990, 29, 1147-1160.

11. Mistrali, F.; Volpin, D.; Garibaldo, G. B.; Ciferri, A. J Phys Chem 1971, 75, 142-149.

12. Li, B.; Daggett, V. J Musc Res Cell Motil 2002, 23, 561-573.

13. Villani, V.; Tamburro, A. M. Ann NY Acad Sci 1999, 879, 284287.

14. Tamburro, A. M.; Pepe, A.; Bochicchio, B.; Quaglino, D.; Ronchetti, I. P. J Biol Chem 2005, 280, 2682-2690.

15. Martino, M.; Coviello, A.; Tamburro, A. M. J Biol Macromol 2000. 27, 59-64.

16. Kumashiro, K. K.; Kurano, T. L.; Niemczura, W.; Martino, M.; Tamburro, A. M. Biopolymers 2003, 70 221-226.

17. Tamburro, A. M.; Pepe, A.; Bochicchio, B. Biochemistry 2006, 45, 9518-9530.

18. Yao, X. L.; McMillian, R. A.; Conticello, V. P.; Hong, M. Magn Reson Chem 2004, 42, 267-275.

19. Fleming, W. W.; Sullivan, C. E.; Torchia, D. A. Biopolymers 1980, 19, 597-617.

20. Perry A.; Stypa, M. P.; Tenn, B. K.; Kumashiro, K. K. Biophys J 2002, 82, 1086-1095.

21. Ellis, G. E.; Packer, K. J. Biopolymers 1976, 13, 813-832.

22. Callaghan, P. T. Principles of Nuclear Magnetic Resonance Microscopy; Oxford University Press, 1991.

23. Mansfield, P.; Grannell, P. K. Phys Rev B 1975, 12, 3618-3634.

24. Mitra, P. P.; Sen, P. N.; Schwartz, L. M. Phys Rev Lett 1992, 68, 3555-3558.

25. Mair, R. W.; Hurlimann, M. D.; Sen, P. N.; Schwartz, L. M.; Patz, S.; Walsworth, R. L. J Magn Reson 2001, 19, 345-351.

26. Zielinski, L. J.; Sen, P. N.; Hurlimann, M. D.; Patz, S.; Cory, D. G.; Walsworth R. L. J Magn Reson 2003, 165, 153-161.

27. Conradi, M. S.; Bruns, M. A.; Sukstanskii, A. L.; Gross, S. S.; Leawoods, J. C. J Magn Reson 2004, 169, 196-202.

28. Latour, L. L.; Svobada, K.; Mitra, P. P.; Sotak, C. H. Proc Natl Acad Sci 1994, 91, 1229-1233.

29. Gotte, L.; Mammi, M.; Pezzin, G. Connect Tissue Res 1972, 1, 61-67.

30. Zhang, W.; Cory, D. G. J Magn Res 1998, 132, 144-149.

31. Yablonskiy, D. A.; Bretthorst, G. L.; Ackerman, J. J. H. Magn Reson Med 2003, 50, 664-669.

32. Gotte, L.; Mammi, M.; Pezzin, G. Connect Tissue Res 1972, 1, 61-67.

33. Latour, L. L.; Svoboda, K.; Mitra, P. P.; Sotak, C. H. Proc Natl Acad Sci 1994, 91, 1229-1233.

Reviewing Editor: C. Bush 